\title{
Distinct genomic profiles of gestational choriocarcinoma, a unique cancer of pregnant tissues
}

\author{
Seung-Hyun Jung ${ }^{1,2}$, Youn Jin Choi ${ }^{3,4}$, Min Sung Kim ${ }^{1,5}$, Hyeon-Chun Park ${ }^{6,7}$, Mi-Ryung Han ${ }^{7,8}$, Soo Young Hur ${ }^{3,4}$, \\ Ah Won Lee ${ }^{9}$, Ok Ran Shin ${ }^{9}$, Jeana Kim ${ }^{9}$, Sung Hak Lee ${ }^{9}$, Dongwan Hong $\mathbb{1 0}^{4}$, Sang Yong Song ${ }^{10}$, \\ Yeun-Jun Chung ${ }^{6,7}$ and Sug Hyung Lee Le $^{1,5}$
}

\begin{abstract}
Little is known about genomic alterations of gestational choriocarcinoma (GC), unique cancer that originates in pregnant tissues, and the progression mechanisms from the nonmalignant complete hydatidiform mole (CHM) to GC. Whole-exome sequencing (20 GCs) and/or single-nucleotide polymorphism microarray (29 GCs) were performed. We analyzed copy-neutral loss-of-heterozygosity (CN-LOH) in 29 GCs that exhibited androgenetic CN-LOHs (20 monospermic, 8 dispermic) and no CN-LOH (one with NLRP7 mutation). Most GCs (25/29) harboring recurrent copy number alterations (CNAs) and gains on 1q21.1-q44 were significantly associated with poor prognosis. We detected five driver mutations in the GCS, most of which were chromatin remodeling gene (ARID1A, SMARCD1, and EP300) mutations but not in common cancer genes such as TP53 and KRAS. One patient's serial CHM/invasive mole/GC showed consistent CN-LOHs, but only the GC harbored CNAs, indicating that $\mathrm{CN}-\mathrm{LOH}$ is an early pivotal event in $\mathrm{HM}$ IM-GC development, and CNAs may be a late event that promotes CHM progression to GC. Our data indicate that GCs have unique profiles of CN-LOHs, mutations and CNAs that together differentiate GCs from non-GCs. Practically, CN-LOH and CNA profiles are useful for the molecular diagnosis of GC and the selection of GC patients with poor prognosis for more intensive treatments, respectively.
\end{abstract}

\section{Introduction}

Gestational trophoblastic diseases (GTDs), including hydatidiform mole (HM), invasive mole (IM), and gestational choriocarcinoma (GC), encompass a spectrum of tumorous conditions featuring proliferation of a woman's pregnant tissues (placental trophoblasts) but not of a woman's own tissues ${ }^{1,2}$. HMs are further subdivided into complete and partial $\mathrm{HMs}^{3}$. Chromosomal polymorphism analyses have identified that most complete HMs (CHMs) arise when an ovum without maternal chromosomes is

Correspondence: Yeun-Jun Chung (yejun@catholic.ac.kr) or

Sug Hyung Lee (suhulee@catholic.ac.kr)

${ }^{1}$ Cancer Evolution Research Center, The Catholic University of Korea, Seoul, Korea

${ }^{2}$ Department of Biochemistry, The Catholic University of Korea, Seoul, Korea Full list of author information is available at the end of the article

These authors contributed equally: Seung-Hyun Jung, Youn Jin Choi fertilized by one sperm that duplicates its DNA (monospermic diploid or uniparental diploid), and only some CHMs $(\sim 10 \%)$ arise from fertilization by two sperm (dispermic diploid) $^{1}$. Both CHM types could produce copy-neutral loss-of-heterozygosity $(\mathrm{CN}-\mathrm{LOH})$ due to errors in meiosis I or meiosis II, which would result in two copies of a chromosome from one parent and no copies from the other parent with no change in the copy number $^{4-6}$. In contrast, partial HM arises from two sperm with an ovum (69XXX, 69XXY, or 69XYY) ${ }^{4-6}$. Many CHMs progress to IM, which invades the uterine wall or blood vessels and rarely metastasizes to distant sites ${ }^{7}$. Eventually, some CHMs ( $\sim 3 \%)$ give rise to GCs, which occupy the malignant end of the GTD spectrum ${ }^{7}$. GCs are known to be preceded by CHMs (50\%), previous abortions (25\%), or normal/ectopic pregnancies $(25 \%)^{1}$. They are highly 
sensitive to chemotherapy, but untreated cases can rapidly metastasize to distant organs and may be fatal ${ }^{1,8}$. Some GCs frequently present with metastasis in the brain or lung even without a diagnosis of uterine $\mathrm{CHM}$ or $\mathrm{GC}^{1,9}$. However, little is known about predictive biomarkers for poor prognosis.

Preneoplastic conditions such as Barrett's esophagus frequently harbor genetic alterations even before the progression to a frank cancer ${ }^{10}$. CHM is considered a preneoplastic condition of GC, and IM has neoplastic characteristics such as tissue invasion ${ }^{1-4}$. However, it is not known what triggers $\mathrm{CHM} / \mathrm{IM}$ to progress to $\mathrm{GC}^{11}$. Genomic alterations of GCs, especially with nextgeneration sequencing (NGS), are not well studied due to the rare availability of tumor tissues (frequent tissue necrosis, infrequent surgery). One study reported wholeexome sequencing (WES) data on a single case of GC, but it did not disclose any well-known driver mutation, probably due to the small case number ${ }^{12}$. To date, cytogenetic analyses of GCs have revealed aneuploidy with a diverse range of chromosomal alterations, including frequent 7p12-q11.2 and 8p12-p21 losses and 7q21-q31 gains $^{13-15}$. An array comparative genomic hybridization study identified frequent gains of $1 \mathrm{p} 36$ and $17 \mathrm{p} 25$ and losses of $9 \mathrm{q} 33,17 \mathrm{q} 21$ and $18 \mathrm{q} 22^{16}$. However, remarkable driver gene mutations have not yet been identified in GCs by gene-to-gene analyses ${ }^{17,18}$. The lack of genome-wide alteration data on $\mathrm{HM} / \mathrm{IM} / \mathrm{GC}$, even in this NGS era, led us to analyze GC genomes by WES, copy number alteration (CNA), and CN-LOH profiles in this study.

\section{Materials and methods}

\section{Hydatidiform mole, invasive mole, and choriocarcinoma} tissues

Formalin-fixed and paraffin-embedded (FFPE) tissues of 31 GTDs in 29 patients (CHM/IM/GC tissues in 1 patient (GC15) and GCs in 28 patients) were used for this study. All of these patients were Korean women, and approval for this study was obtained from the Institutional Review Board at The Catholic University of Korea, College of Medicine (KC16TISE0342). The diagnosis of GC was made based on the clinical or histopathologic criteria of the International Federation of Gynecology and Obstetrics $(\mathrm{FIGO})^{7}$. Histologically, GC is a malignancy characterized by trophoblast proliferation, absence of chorionic villi, and tissue necrosis with bleeding, which were confirmed by two pathologists under microscopic examination. The 29 GCs consisted of 21 primary GCs in the uterus or fallopian tube and eight metastatic GCs (six in the lungs, one in the rectum, and one in the brain). Pregnancy-related history was available in 25 patients (14 with CHMs and 11 with nonmolar pregnancies [six with abortion, one with ectopic pregnancy, and four with term pregnancy]) but was not available in four patients. The clinicopathologic features of the cases are summarized in Table 1. CHM, IM, and GC cells as well as matched normal cells in each case were procured from hematoxylin and eosin-stained slides using microdissection by a board-certified pathologist, as described previously ${ }^{19}$. Tumor cell purities were $\sim 50-80 \%$, which were confirmed by two pathologists under microscopic examination. For genomic DNA extraction, the DNeasy Blood \& Tissue Kit (Qiagen) was used. None of the presumed partners' DNA was available for analyses.

\section{Short tandem repeat (STR) marker analysis}

To identify the genetic sources of GTDs, STR analysis was performed using genomic DNA from GTD tissues and matched normal cells using the AmpFLSTR Identifiler Plus PCR Amplification Kit (Thermo Fisher Scientific) that covered 16 STR loci (polymorphic DNA markers): D8S1179, D21S11, D7S820, CSF1PO, D3S1358, TH01, D13S317, D16S539, D2S1338, D19S433, vWA, TPOX, D18S51, Amelogenin, D5S818, and FGA. The fluorescent PCR products were analyzed using the ABI 3130XL Genetic Analyzer (Applied Biosystems). Each fluorescent peak was quantified by its size (base pairs), peak height, and peak area, as previously reported ${ }^{15}$, and analyzed by GeneMapper 4.1 software (Applied Biosystems).

\section{Copy number alteration and loss-of-heterozygosity analyses}

Both CNA and LOH of the 29 GTDs were analyzed using a SNP microarray with an Affymetrix OncoScan FFPE assay (Affymetrix) according to the manufacturer's instructions. OncoScan OSCHP files processed by the TuScan algorithm and the SNP-FASST2 segmentation algorithm in NEXUS software v9.0 (BioDiscovery) were used to define CNAs and LOHs for each sample ${ }^{20}$. Segments were classified as copy gains and losses when the $\log _{2}$ ratio was $>0.2$ and $<-0.2$, respectively. A homozygous value larger than 0.7 was defined as $\mathrm{LOH}$. B-allele frequencies could be attenuated depending on the levels of normal cell contamination from patients (Supplementary Table 1), which was considered in the interpretation.

\section{WES analysis}

WES libraries were constructed using the Agilent SureSelect Human All Exome $50 \mathrm{Mb}$ kit (Agilent Technologies) according to the manufacturer's instructions. Input quantities of genomic DNA were equally adjusted for WES analyses. Per WES reaction, we used $800 \mathrm{ng}$ of double-stranded genomic DNA each for normal, CHM, IM, and GC samples. WES libraries were sequenced using the Illumina HiSeq2000 platform to generate 101-bp paired-end reads. Burrows-Wheeler Aligner was used to align the sequencing reads to the human reference 
Table 1 Clinicopathologic characteristics of 29 choriocarcinoma patients.

\begin{tabular}{|c|c|c|c|c|c|c|c|}
\hline Case & Age & Diagnosis & Tissues analyzed ${ }^{a}$ & Antecedent pregnancy & LOH pattern ${ }^{\mathbf{b}}$ & STR $^{c}$ & Survival status ${ }^{\mathrm{d}}$ \\
\hline GC01 & 23 & Choriocarcinoma & Uterus & $\mathrm{CHM}$ & Monospermic & Single nonmaternal peak & Poor survival \\
\hline GCO2 & 33 & Choriocarcinoma & Uterus & Term pregnancy & Biparental & N/A & Good survival \\
\hline GCO3 & 37 & Choriocarcinoma & Uterus & $\mathrm{CHM}$ & Monospermic & N/A & Poor survival \\
\hline GCO4 & 31 & Choriocarcinoma & Uterus & $\mathrm{CHM}$ & Monospermic & Single nonmaternal peak & Good survival \\
\hline GC05 & 30 & Choriocarcinoma & Uterus & Term pregnancy & Monospermic & Single nonmaternal peak & Good survival \\
\hline GC06 & 48 & Choriocarcinoma & Uterus & $\mathrm{CHM}$ & Monospermic & N/A & Good survival \\
\hline GCO7 & 39 & Choriocarcinoma & Uterus & $\mathrm{CHM}$ & Monospermic & Single nonmaternal peak & Good survival \\
\hline GC08 & 26 & Choriocarcinoma & Uterus & Ectopic pregnancy & Dispermic & Double nonmaternal peak & Poor survival \\
\hline GC09 & 46 & Choriocarcinoma & Uterus & Abortion & Monospermic & Single nonmaternal peak & Good survival \\
\hline GC10 & 42 & Choriocarcinoma & Uterus & Abortion & Monospermic & Single nonmaternal peak & Good survival \\
\hline GC11 & 52 & Choriocarcinoma & Uterus & Abortion & Monospermic & Single nonmaternal peak & Good survival \\
\hline GC12 & 51 & Choriocarcinoma & Uterus & $\mathrm{CHM}$ & Dispermic & Double nonmaternal peak & Good survival \\
\hline GC13 & 39 & Choriocarcinoma & Lung & Abortion & Dispermic & Double nonmaternal peak & Poor survival \\
\hline GC14 & 43 & Choriocarcinoma & Lung & Abortion & Monospermic & Single nonmaternal peak & Good survival \\
\hline GC15 & 54 & Choriocarcinoma & Brain & $\mathrm{CHM}$ & Monospermic & Single nonmaternal peak & Poor survival \\
\hline GC16 & 68 & Choriocarcinoma & Uterus & $\mathrm{CHM}$ & Dispermic & Double nonmaternal peak & Good survival \\
\hline GC17 & 48 & Choriocarcinoma & Fallopian tube & Unknown & Monospermic & Single nonmaternal peak & Good survival \\
\hline GC18 & 34 & Choriocarcinoma & Lung & $\mathrm{CHM}$ & Monospermic & Single nonmaternal peak & Poor survival \\
\hline GC19 & 35 & Choriocarcinoma & Uterus & $\mathrm{CHM}$ & Monospermic & Single nonmaternal peak & Good survival \\
\hline GC20 & 43 & Choriocarcinoma & Uterus & Unknown & Monospermic & Single nonmaternal peak & Good survival \\
\hline GC21 & 58 & Choriocarcinoma & Rectum & Unknown & Monospermic & Single nonmaternal peak & Good survival \\
\hline GC22 & 33 & Choriocarcinoma & Uterus & $\mathrm{CHM}$ & Dispermic & N/A & Poor survival \\
\hline GC23 & 32 & Choriocarcinoma & Uterus & $\mathrm{CHM}$ & Monospermic & Single nonmaternal peak & Poor survival \\
\hline GC24 & 28 & Choriocarcinoma & Lung & Unknown & Monospermic & Single nonmaternal peak & Poor survival \\
\hline GC25 & 31 & Choriocarcinoma & Lung & Term pregnancy & Dispermic & Double nonmaternal peak & Good survival \\
\hline GC26 & 34 & Choriocarcinoma & Uterus & Abortion & Dispermic & N/A & Good survival \\
\hline GC27 & 57 & Choriocarcinoma & Lung & $\mathrm{CHM}$ & Monospermic & Single nonmaternal peak & Good survival \\
\hline GC28 & 39 & Choriocarcinoma & Uterus & Term pregnancy & Dispermic & Single nonmaternal peak & Poor survival \\
\hline GC29 & 45 & Choriocarcinoma & Uterus & $\mathrm{CHM}$ & Monospermic & Single nonmaternal peak & Poor survival \\
\hline
\end{tabular}

CHM complete hydatidiform mole, $\mathrm{LOH}$ loss of heterozygosity, N/A not available.

${ }^{\text {aPrimary }}$ cancer: the uterus and fallopian tube, metastatic cancer: lung, rectum, and brain.

${ }^{b} \mathrm{LOH}$ pattern was determined using SNP microarray.

'STR markers were analyzed using the AmpFLSTR PCR Amplification Kit.

'The patients who survived $<5$ years after GC diagnosis were considered "poor survival", and those who survived $>5$ years after GC diagnosis were considered "good survival".

genome (hg19). Processing and management of sequencing data were performed as described elsewhere ${ }^{21}$. In brief, somatic genomic variants were identified using $\mathrm{MuTect}^{22}$ and SomaticIndelDetector ${ }^{23}$ for point mutations and indels, respectively. The ANNOVAR package was used to select somatic variants located in coding sequences and predict their functional consequences, such as silent or nonsilent variants ${ }^{24}$. To identify somatic mutations in GTD, WES was performed in 22 cases (20 GCs, $1 \mathrm{CHM}$, and $1 \mathrm{IM})$. WES was not available for the other 9 cases due to low quality. To obtain reliable and robust mutation calling, the following somatic variants were eliminated: (i) read depth $<20$ in either tumor or matched normal tissue; (ii) any polymorphisms referenced in either the 1000 Genomes Project or Exome Aggregation Consortium with a minor allele frequency of $1 \%$ or 
more in East Asians; and (iii) polymorphisms that showed $>1 \%$ minor allele frequency in our in-house normal database (397 whole-genome sequencing data from Korean populations). Because the majority of variants detected in GCs were likely to be paternal polymorphisms ${ }^{1,2}$, they were stringently filtered by dbSNP (version 147). Subsequently, variants reported in the clinically relevant variant (ClinVar) database were rescued to prevent the exclusion of true pathogenic variants.

\section{Results}

\section{Identification of GC origins}

Since GCs are known to arise from androgenetic CHMs harboring $\mathrm{CN}-\mathrm{LOH}^{16}$, we first attempted to address the origins of the GCs. For this, the CN-LOH patterns of 29 GCs were analyzed by single-nucleotide polymorphism (SNP) microarray, which identified three different B-allele patterns (monospermic, dispermic, and biparental). The $\mathrm{B}$-allele frequency (BAF) is calculated by dividing the number of minor (B) alleles by the sum of major (A) and minor alleles. BAF can have values of 100\% (BB allele), $50 \%$ ( $\mathrm{AB}$ allele), and $0 \%$ (AA allele) in a diploid individual. In $\mathrm{CN}-\mathrm{LOH}$, a two-track BAF plot (100 and 0\%) representing one haplotype was observed without copy number change. From this point of view, twenty GCs (69\%) showed a monospermic pattern (Table 1 and Supplementary Fig. 1) that displayed simple LOHs with only two haplotypes (0 and 100\%) across the entire genome (Fig. 1a-c). Another 8 GCs (28\%) were identified as the dispermic pattern that showed LOHs across the entire genome with intermittent additional haplotypes (50\%), which might be an oscillation of two-haplotype status reflecting the haplotype composition of each sperm (Fig. 1d and Supplementary Fig. 1). The diploidic B-allele pattern of the other GC (GC02) (Fig. 1e) and abundant SNP mismatches between this GC and the patient's normal cells $(n=2353)$ suggested its nonandrogenetic gestational origin. This patient had a heterozygous NLRP7 germline mutation (p.Y209C). Very rarely, nonandrogenetic biparental CHMs develop in the family with NLRP7 germline mutations ${ }^{25}$. Unfortunately, however, her history of molar pregnancy was not available in the hospital record. STR analyses using GCs and matched normal tissues corresponded to those of the B-allele patterns (the monospermic patterns in Fig. $1 \mathrm{a}-\mathrm{c}$ and Supplementary Table 2; the dispermic patterns in Fig. 1d and Supplementary Table 2).

\section{Copy number alterations}

A total of 185 CNAs (109 gains and 76 losses) were identified in the 29 GCs (Fig. 2a and Supplementary Table 3), corresponding to a median $7.4 \%$ fraction of the genome altered (FGA). These results are similar to the rate of uterine endometrial carcinoma ( $7.2 \%$ FGA) but lower than those observed in nongestational germ cell tumors in the testis (37.3\% FGA) and other cancers (Supplementary Fig. 2a). Next, we compared our CNA calls from the SNP array with those from WES using the ngCGH module in NEXUS software. The concordance level was estimated by calculating the ratio of overlapping lengths of alterations against total lengths of alterations between the SNP array and WES. We identified that the concordance between them reached $93.6 \%$ (range, 79.8-99.9\%), suggesting that the CNAs estimated by two independent platforms were in agreement (Supplementary Fig. 3). A majority of the GCs $(n=25$, 86.2\%) harbored at least two CNAs (median of 5 CNAs, range 2-22), whereas four GCs (GC10, 11, 20, and 27) did not harbor any CNAs. Of note, the poor survival group ( $<5$-year survival from GC diagnosis) harbored significantly higher numbers of CNAs than the good survival group ( $>5$-year survival from $\mathrm{GC}$ diagnosis) (median of 12.0 vs 3.0 CNAs, $P=0.001$ ). There was no significant difference in the number of CNAs between monospermic and dispermic GCs, metastatic and nonmetastatic GCs, or chemotherapy-treated and chemotherapy-naive GCs $(P>0.05)$.

Of the CNAs detected, eight CNA regions (six gains and two losses) were recurrently identified (>6 GCs, 20\%) (Fig. 2b and Supplementary Table 4). Compared to other cancers, in which both gains and losses occur at similar rates, GCs harbor more gains than losses (Supplementary Fig. 2b). The most recurrent CNA was an arm-level copy gain on 1q21.1-q44 (44.8\%). Arm-level copy gain on 12p13.33-p11.1, where KRAS resides, was also detected recurrently (20.7\%). The most well-known CNAs for GCs, a gain on 7q21-q31 and a loss on 8p21-p12, were also detected but were not the most common CNAs in our study. Of note, copy gains on 1q21.1-q44 were significantly associated with poor survival $(P=0.003)$. In addition, copy gains on 9q21.11-q34.3 and 21q21.3-q22.3 were associated with metastasis $(P=0.015)$, and chromosome $X$ deletions (p22.33-p11.1 and q11.1-q28) were associated with dispermic origin $\left(P=1.4 \times 10^{-5}\right)$. The frequent chromosome $\mathrm{X}$ deletions in the dispermic origin could be explained by the tumor originally being the $\mathrm{XY}$ chromosome.

\section{Mutation profiles}

Due to the low quality of GC DNA, not all cases (20 of the 29 GCs) were analyzed by WES (107X (range 49-154X) for GC tissues and 122X (range 55-150X) for matched normal tissues) (Supplementary Table 5). A total of 10,964 nonsilent mutations $(10,707$ SNVs and 257 indels) were identified by applying stringent germline variant filter criteria (Supplementary Table 6). The number of mutations was significantly enriched in GCs with a history of chemotherapy $(P=0.036)$, and 2 GCs 


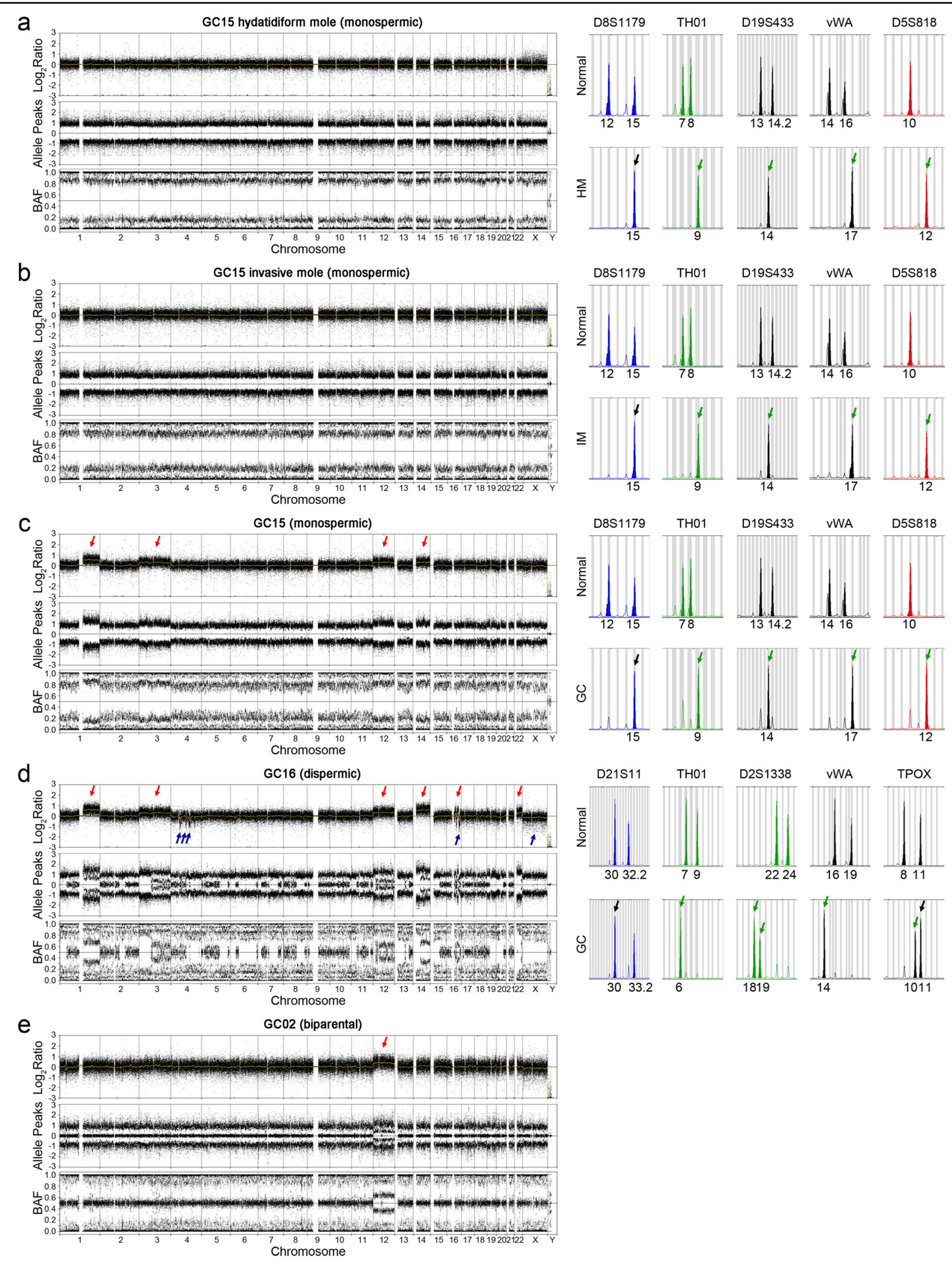

Fig. 1 (See legend on next page.) 
(see figure on previous page)

Fig. 1 Representative patterns of gestational choriocarcinoma genomes. Monospermic (a-c), dispermic (d), and biparental (e) patterns are featured by SNP microarray (left column) and STR marker analysis (right). One patient (GC15) had serial CHM, IM, and GC, and each of these tissues was analyzed (a-c). In the SNP microarray, logR ratio plots (upper), allele peak plots (middle), and B-allele frequency (BAF) plots (bottom) are shown. Red and blue arrows in the logR ratio plot represent the copy number gain and loss, respectively. Green and black arrows in the STR marker analysis represent the informative alleles that are not identified in matched normal and noninformative alleles, respectively. BAF plots of GC15 (CHM, IM and $\mathrm{GC}$ ) and GC16 represent two tracks (BB allele (100\%) and AA allele (0\%)) without the AB allele (50\%), suggesting CN-LOH.
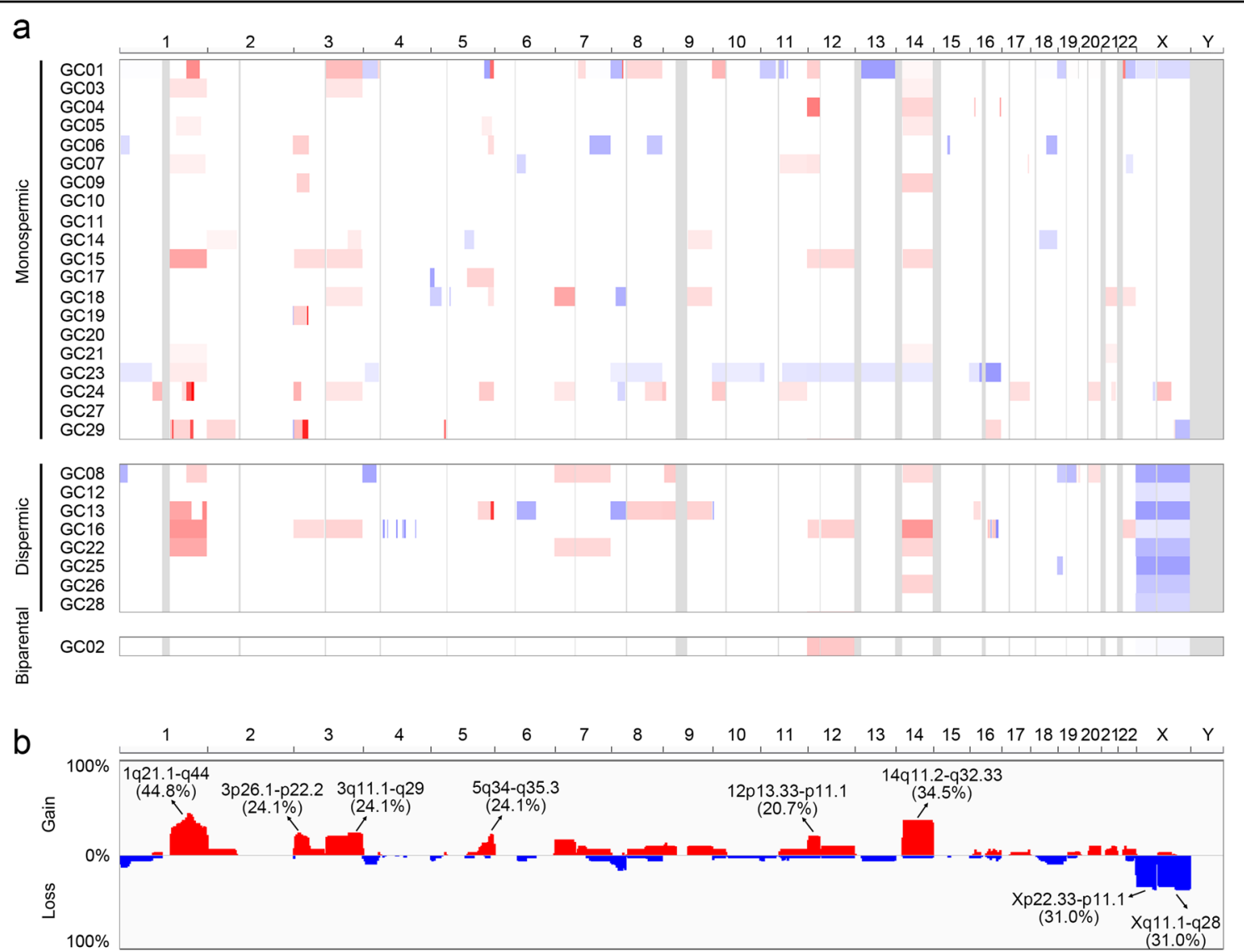

Fig. 2 Copy number profiles of GCs. a Heat map showing the chromosomal copy gains (red) and losses (blue) in each sample. Boundaries of individual chromosomes are indicated by vertical bars. b Frequencies (y-axis) of copy number gains and losses across the whole genomes of GC genomes. Red denotes copy number gains, and blue denotes copy number losses.

(GC08 and 13) obtained after chemotherapy exhibited exceptionally high numbers of mutations. Of the mutated genes detected in our study, nine genes, including ARID1A (p.K1382*), SMARCD1 (p.R303*), EP300 (p.G1999R), AMER1 (p.R353Q), and ZNF429 (p.K568E) have been reported in the COSMIC database and could be considered driver mutations (Fig. 3). Mutations in ARID1A, SMARCD1, and ZNF429 were either hotspot mutations (ARID1A and ZNF429) or had functional relevance (inactivating mutations in tumor suppressor SMARCD1) (https://cancer.sanger.ac.uk/ cosmic/mutation $)^{26}$. All of the driver mutations were successfully validated with either digital PCR or Sanger sequencing (Supplementary Fig. 4). These data show that most well-known driver genes in solid cancers, including TP53 and KRAS, may not be common in GCs, but other genes, such as those involved in chromatin remodeling (ARID1A, SMARCD1, and EP300), may represent mutational drivers in GCs.

\section{Sequential analyses in one patient; hydatidiform mole- invasive mole-choriocarcinoma}

One patient (GC15) had consecutive CHM, IM, and GC development, each of which was studied in this study using SNP microarray, STR markers, and WES. The CHM harbored CN-LOH with the two-track BAF plot (100 and 


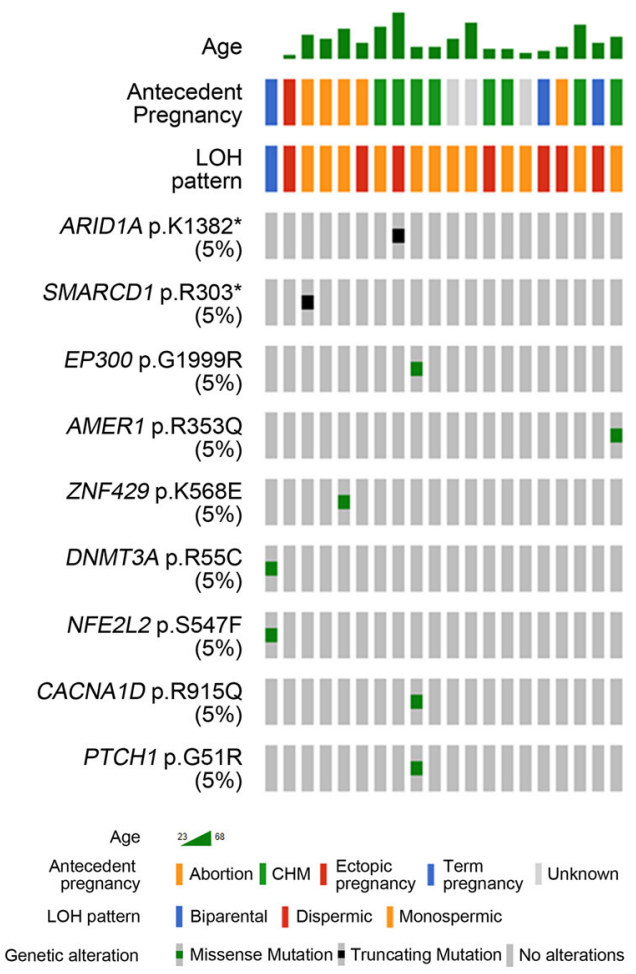

Fig. 3 Cancer-related mutations in the GC genomes. Each row represents the mutated gene, and each column represents an individual patient.

$0 \%$ in the SNP array across the whole genome, indicating a monospermic pattern (Fig. 1a). This was confirmed by the STR assay, which showed a single nonmaternal peak for all informative alleles (Fig. 1a and Supplementary Table 2). Both SNP and STR assays also revealed constant patterns (monospermic) among the IM and GC (Fig. 1b, c). Of note, CNAs were evident in GC (Fig. 1c; copy gains on 1q, 3, 12, and 14q) but not in CHM or IM. The CHM, IM, and GC of this patient harbored similar numbers of nonsilent somatic mutations (48, 45, and 57 mutations, respectively) (Supplementary Table 6) without any driver mutations. These results suggest that CNA may play a critical role in $\mathrm{HM} / \mathrm{IM}$ progression to $\mathrm{GC}$.

\section{Discussion}

Our analyses of GC genomes had two goals. First, we attempted to disclose the genomic alteration profiles of GCs (CN-LOH, CNA, and somatic mutation) that drive tumorigenesis. Second, we wanted to identify any differences in genomic alterations of GCs from those of common (i.e., GC vs. nongestational) cancers. Our data show that CNAs are common genomic alterations in GCs, suggesting that most GCs may occur on the basis of $\mathrm{CN}$ LOH-harboring $\mathrm{CHM}$ that may progress to $\mathrm{GC}$ by accumulating CNAs. We identified some driver mutations in
GCs, but none of them were recurrent. Together, our results indicate that genomic alteration profiles of GCs are different from those of nongestational cancers and suggest that recurrent CNAs appear to be drivers of GC development.

Compared to the reports of $\mathrm{HM}$ history to GC $(27-80 \%)^{27}, \mathrm{CN}-\mathrm{LOH}$ in our study $(96.6 \%)$ was relatively high. There could be several possible explanations for this disagreement. First, earlier studies to find a causative type of pregnancy might depend on patients' history but not on pathologic examination, which could underestimate molar pregnancies ${ }^{28}$. In our data, $10 \mathrm{GCs}$ without a history of molar pregnancies but with term/ectopic/abortion pregnancies showed monospermic or dispermic $\mathrm{CN}$ LOH. Second, the discrepancy could arise from sampling bias because our sample size was relatively small.

One GC without any CN-LOH exhibited a diploidic Ballele pattern with abundant SNP mismatches with the patient's genome, strongly suggesting its biparental origin. This patient had a heterozygous germline mutation in the NLRP7 gene. However, the NLRP7 mutation was a novel variant that had not been reported previously (OMIM ID: 609661). In addition, based on the notion that only biallelic mutations in NLRP7 have been associated with recurrent biparental $\mathrm{HMs}^{25}$, this heterozygous mutation may not be associated with GC development. This GC showed no discernible difference in CNAs except a copy gain on chromosome 12 .

The present study discovered novel CNAs (gains on 1q, 3p26.1-p22.2, 3q11.1-q29, 5q34-q35.3, 12p, and 14q) as well as previously known CNAs (7q21-q31 gain and 8p21p12 loss) in GCs. Despite the high sensitivity to chemotherapy for GC, distant metastasis is still an indicator for poor survival ${ }^{1}$. In the present study, we discovered that CNAs might be a biomarker for both poor survival and metastasis of GCs. To our knowledge, our data are the first molecular data for GC prognosis.

GC is a bona fide cancer, but definite cancer genes for GCs are not known $^{18}$, suggesting that GC mutations might not occur in well-known cancer genes. By NGSbased WES, we discovered that GCs harbored a small number of driver mutations, which included chromatin remodeling genes. Our results indicate that the contribution of somatic mutations to GC development may be different in quantity (driver mutation numbers) and quality (mutated gene functions) compared to other cancers. Recurrent inactivating mutations or losses/deletions of ARID1A have been found in many cancers (https://cancer.sanger.ac.uk/cosmic/mutation) ${ }^{29}$, suggesting the role of ARID1A as a tumor suppressor gene (TSG). In addition to an ARID1A frameshift mutation in one case (Fig. 3), another GC (GC06 and 23) showed copy losses at the ARID1A locus (2/29 GCs, Fig. 2a), suggesting that ARID1A inactivation by genetic alterations might be a 
common event in GCs (3/29, 10.3\%). ZNF429 encodes a transcription factor ${ }^{30}$, but its exact role in cancer pathogenesis remains unknown. The ZNF429 p.K568E mutation detected in our study is the second most common variant (35/312 missense mutations) in the COSMIC database and has been identified solely in glioblastomas (https://cancer.sanger.ac.uk). SMARCD1 interacts with $\mathrm{p} 53$, and their uncoupling results in inhibition of p53dependent apoptosis and cell cycle arrest, suggesting that SMARCD1 is a putative $\mathrm{TSG}^{31}$. An inactivating mutation of SMARCD1 (p.R303*) detected in the present study might inactivate the TSG functions of SMARCD1. Of note, mutations in ARID1A, SMARCD1, and EP300 detected in the present study are chromatin remodeling genes $^{31}$, suggesting that alterations of chromatin remodeling might be involved in GC tumorigenesis.

In sequential samples (HM-IM-GC), we found evidence that GTDs might be clonal with a constant $\mathrm{CN}$ LOH pattern. Neither the HM nor IM in this patient harbored CNAs, whereas the GC harbored several CNAs but not driver mutations. Taken together, these findings indicate that $\mathrm{CN}-\mathrm{LOH}$ is an early pivotal event in HM-IM-GC development and that CNA is a late event that may promote $\mathrm{HM} / \mathrm{IM}$ progression to GC. In addition, common genomic profiles between the $\mathrm{HM}$ and IM (constant CN-LOH, no CNAs, and no driver mutations) suggest that the progressed phenotype of IM (invasion and dissemination) might be caused by nongenetic factors.

The present study identified integrative genetic characteristics of GCs for the first time. We also evaluated genetic alterations in relation to clinical data and found that CNAs could be an important factor for disease progression or for poor prognosis in GCs. The present study provides clues for understanding the etiology of GC, properly diagnosing HM-IM-GC, and selecting patients with $\mathrm{GC}$ who might require intensive treatments against poor prognosis.

\section{Acknowledgements}

This study was supported by grants from the National Research Foundation of Korea (2017R1A2B2002314, 2019R1A5A2027588, 2017R1E1A1A01074913, and 2019R1C1C1004909).

\footnotetext{
Author details

${ }^{1}$ Cancer Evolution Research Center, The Catholic University of Korea, Seoul, Korea. ${ }^{2}$ Department of Biochemistry, The Catholic University of Korea, Seoul, Korea. ${ }^{3}$ Department of Obstetrics and Gynecology, The Catholic University of Korea, Seoul, Korea. ${ }^{4}$ Cancer Research Institute, The Catholic University of Korea, Seoul, Korea. ${ }^{5}$ Department of Pathology, The Catholic University of Korea, Seoul, Korea. ${ }^{6}$ Integrated Research Center for Genome Polymorphism, The Catholic University of Korea, Seoul, Korea. ${ }^{7}$ Department of Microbiology, The Catholic University of Korea, Seoul, Korea. ${ }^{8}$ Division of Life Sciences, College of Life Sciences and Bioengineering, Incheon National University, Incheon, Korea. ${ }^{9}$ Department of Hospital Pathology, College of Medicine, The Catholic University of Korea, Seoul, Korea. ${ }^{10}$ Department of Pathology and Translational Genomics, Samsung Medical Center, Sungkyunkwan University School of Medicine, Seoul, Korea
}

\section{Data availability}

The datasets supporting the conclusions of this articleare included within the article and its additional files. Rawdata (sequences) were deposited in the SRA database(Project ID: PRJNA636516).

\section{Conflict of interest}

The authors declare that they have no conflict of interest.

\section{Publisher's note}

Springer Nature remains neutral with regard to jurisdictional claims in published maps and institutional affiliations.

Supplementary information accompanies this paper at https://doi.org/ 10.1038/s12276-020-00544-0.

Received: 26 July 2020 Revised: 4 November 2020 Accepted: 4 November 2020.

Published online: 15 December 2020

\section{References}

1. Seckl, M. J., Sebire, N. J. \& Berkowitz, R. S. Gestational trophoblastic disease. Lancet 376, 717-729 (2010).

2. Froeling, F. E. \& Seckl, M. J. Gestational trophoblastic tumours: an update for 2014. Curr. Oncol. Rep. 16, 408 (2014).

3. Hui, P., Martel, M. \& Parkash, V. Gestational trophoblastic diseases: recent advances in histopathologic diagnosis and related genetic aspects. Adv. Anat. Pathol. 12, 116-125 (2005).

4. Kajii, T. \& Ohama, K. Androgenetic origin of hydatidiform mole. Nature $\mathbf{2 6 8}$ 633-634 (1977).

5. Bifulco, C. et al. Genotypic analysis of hydatidiform mole: an accurate and practical method of diagnosis. Am. J. Surg. Pathol. 32, 445-451 (2008).

6. McConnell, T. G., Murphy, K. M., Hafez, M., Vang, R. \& Ronnett, B. M. Diagnosis and subclassification of hydatidiform moles using p57 immunohistochemistry and molecular genotyping: validation and prospective analysis in routine and consultation practice settings with development of an algorithmic approach. Am. J. Surg. Pathol. 33, 805-817 (2009).

7. Lurain, J. R. Gestational trophoblastic disease II: classification and management of gestational trophoblastic neoplasia. Am. J. Obstet. Gynecol. 204, 11-18 (2011).

8. Berkowitz, R. S. \& Goldstein, D. P. Current management of gestational trophoblastic diseases. Gynecol. Oncol. 112, 654-662 (2009).

9. Cole, L. A. et al. Gestational trophoblastic diseases: 2. Hyperglycosylated hCG as a reliable marker of active neoplasia. Gynecol. Oncol. 102, 151-159 (2006).

10. Stachler, M. D. et al. Paired exome analysis of Barrett's esophagus and adenocarcinoma. Nat. Genet. 47, 1047-1055 (2015).

11. Gupta, M. et al. Diagnostic reproducibility of hydatidiform moles: ancillan techniques (p57 immunohistochemistry and molecular genotyping) improve morphologic diagnosis for both recently trained and experienced gynecologic pathologists. Am. J. Surg. Pathol. 36, 1747-1760 (2012).

12. Lazare, C. et al. A pilot study comparing the genetic molecular biology of gestational and non-gestational choriocarcinoma. Am. J. Transl. Res. 11, 7049-7062 (2019).

13. Surti, U. \& Habibian, R. Chromosomal rearrangement in choriocarcinoma cell lines. Cancer Genet. Cytogenet. 38, 229-240 (1989).

14. Ahmed, M. N., Kim, K., Haddad, B., Berchuck, A. \& Qumsiyeh, M. B. Comparative genomic hybridization studies in hydatidiform moles and choriocarcinoma: amplification of 7q21-q31 and loss of 8p12-p21 in choriocarcinoma. Cancer Genet. Cytogenet. 116, 10-15 (2000).

15. Matsuda, T. et al. Human chromosome 7 carries a putative tumor suppressor gene(s) involved in choriocarcinoma. Oncogene 15, 2773-2781 (1997).

16. Mello, J. B. et al. Genomic profile in gestational and non-gestational choriocarcinomas. Placenta 50, 8-15 (2017).

17. Vogelstein, B. et al. Cancer genome landscapes. Science 339, 1546-1558 (2013).

18. Li, H. W. Tsao, S. W. \& Cheung, A. N. Current understandings of the molecular genetics of gestational trophoblastic diseases. Placenta $\mathbf{2 3}, 20-31$ (2002). 
19. Lee, J. Y. et al. A simple, precise and economical microdissection technique for analysis of genomic DNA from archival tissue sections. Virchows Arch. $\mathbf{4 3 3}$ 305-309 (1998).

20. Jung, S. H. et al. Whole-exome sequencing identifies recurrent AKT1 mutations in sclerosing hemangioma of lung. Proc. Natl Acad. Sci. USA 113, 10672-10677 (2016).

21. Ashburner, M. et al. Gene ontology: tool for the unification of biology. The Gene Ontology Consortium. Nat. Genet. 25, 25-29 (2000).

22. Cibulskis, K. et al. Sensitive detection of somatic point mutations in impure and heterogeneous cancer samples. Nat. Biotechnol. 31, 213-219 (2013).

23. DePristo, M. A. et al. A framework for variation discovery and genotyping using next-generation DNA sequencing data. Nat. Genet. 43, 491-498 (2011).

24. Wang, K. Li, M. \& Hakonarson, H. ANNOVAR: functional annotation of genetic variants from high-throughput sequencing data. Nucleic Acids Res. 38, e164 (2010).
25. Slim, R. \& Wallace, E. P. NLRP7 and the genetics of hydatidiform moles: recent advances and new challenges. Front. Immunol. 4, 242 (2013).

26. Futreal, P. A. et al. A census of human cancer genes. Nat. Rev. Cancer 4 177-183 (2004)

27. Baltazar, J. C. Epidemiological features of choriocarcinoma. Bull. World Health Organ. 54, 523-532 (1976)

28. Flam, F. \& Rutqvist, L. E. Under-registration of gestational trophoblastic disease in the Swedish Cancer Registry. Eur. J. Epidemiol. 8, 683-686 (1992).

29. Mathur, R. ARID1A loss in cancer: towards a mechanistic understanding. Pharmacol. Therapeut. 190, 15-23 (2018).

30. Stein, W. The ages of the cancer-associated genes. Semin. Oncol. 46, 10-18 (2019).

31. Guan, B., Wang, T.-L. \& Shih, I.-M. ARID1A, a factor that promotes formation of SWI/SNF-mediated chromatin remodeling, is a tumor suppressor in gynecologic cancers. Cancer Res. 71, 6718-6727 (2011). 